\title{
Diagnóstico de laboratorio de COVID-19
}

\author{
Pablo López ${ }^{\star}$, Raquel Ballestét, Verónica Seija ${ }^{\ddagger}$
}

\section{Resumen}

Más de 500 test o pruebas diagnósticas para COVID-19 se están comercializando en el mercado mundial o se encuentran en avanzada fase de desarrollo. En esta situación, sin precedentes, es importante comprender el tipo de ensayos disponibles así como su rol en el proceso diagnóstico. El diagnóstico preciso es clave en el manejo de la pandemia. Esto permite la adecuada identificación de los casos, lo cual habilita las medidas posteriores de control: búsqueda de contactos y aislamiento. En esta revisión describiremos el fundamento y la utilidad de los diferentes tipos de pruebas para diagnóstico etiológico disponibles. También estableceremos las condiciones preanalíticas necesarias para su realización, la sensibilidad y especificidad clínicas, así como la correcta interpretación y emisión de los resultados.

Palabras clave: Coronavirus Diagnóstico Infecciones por coronavirus Técnicas de laboratorio clínico COVID-19

$\begin{array}{ll}\text { Key words: } & \text { Coronavirus } \\ & \text { Diagnosis } \\ & \text { Coronavirus infections } \\ & \text { Clinical laboratory techniques } \\ & \text { Covid-19 }\end{array}$

\section{Introducción}

Testeo, testeo, testeo, ha sido la recomendación de la Organización Mundial de la Salud (OMS) frente a la pandemia de COVID-19. Más de 500 test o pruebas diagnósticas se están comercializando en el mercado mundial o se encuentran en avanzada fase de desarro$11 o^{(1)}$. La falta de un estándar de oro, el uso de diferentes métodos de recolección y preparación de muestras, y una comprensión, aún incompleta, de la dinámica viral en el curso de la infección, dificultan la evaluación rigurosa de la precisión diagnóstica de los innumerables ensayos de SARS-CoV-2 recientemente introducidos ${ }^{(2)}$.

En esta situación, sin precedentes, es importante comprender el tipo de ensayos disponibles así como su rol en el proceso diagnóstico. Y lo más crítico, en términos de la seguridad del paciente, es no asumir que todos los ensayos son siempre precisos en este escenario de una infección emergente ${ }^{(3)}$.

Cuando hablamos de COVID-19 debemos manejar tres premisas:

1. estamos frente a un virus de muy reciente descripción,

2. se trata de una pandemia sin precedentes en los tiempos modernos y

3. estamos trabajando en territorio desconocido y con bibliografía que, muchas veces y hasta este momento, se publica sin revisión de pares ${ }^{(3)}$.

\section{Metodología}

Para la elaboración del presente trabajo revisamos la bibliografía publicada en formato web y disponible en PubMed, o en los portales de cada publicación. También tomamos en cuenta las recomendaciones realizadas por los siguientes organismos: Organización Mundial de la Salud (OMS), Organización Panamericana de la Salud (OPS), Centers for Disease Control and Prevention (CDC), European Centre for Disease Preven-

\footnotetext{
* Profesor adjunto Depto. de Laboratorio de Patología Clínica. Hospital de Clínicas. Facultad de Medicina. † Profesora Depto. de Laboratorio de Patología Clínica. Hospital de Clínicas. Facultad de Medicina. ‡ Profesora agregada Depto. de Laboratorio de Patología Clínica. Hospital de Clínicas. Facultad de Medicina. Correspondencia: Verónica Seija. Correo electrónico: vseija1@gmail.com

Los autores no presentan conflicto de intereses.

Recibido: $26 / 6 / 20$

Aprobado: $7 / 9 / 20$
} 
tion and Control (ECDC), Foundation for Innovative New Diagnostics (FIND), Sociedad Española de Enfermedades Infecciosas y Microbiología Clínica (SEIMC) y Ministerio de Salud Pública del Uruguay (MSP). Se consideraron las publicaciones realizadas hasta el 30 de junio de 2020.

\section{Desarrollo y discusión}

\section{Concepto de sensibilidad y especificidad analítica y clínica}

Vamos a abordar los conceptos de sensibilidad y especificidad analíticas así como los de sensibilidad y especificidad clínicas. La sensibilidad analítica es la cantidad más pequeña de sustancia que se puede detectar en una muestra, de manera confiable, mediante un ensayo (límite de detección). En términos prácticos, para medir la sensibilidad analítica de la prueba molecular para SARS CoV-2 se determina la cantidad mínima de ácido ribonucleico (ARN) que la prueba detectará 95 de cada 100 veces. La especificidad analítica es la capacidad del ensayo para detectar sólo la sustancia deseada en una muestra sin reacción cruzada con otras sustancias. Sin embargo, estas definiciones difieren con los conceptos de sensibilidad y especificidad clínica (el porcentaje de pacientes enfermos que dan positivo y no enfermos que tienen resultados negativos, respectivamente). Una prueba con buena sensibilidad y especificidad analítica no necesariamente tiene buena sensibilidad y especificidad clíni$\mathrm{ca}^{(4)}$. Lamentablemente, el rendimiento clínico de las pruebas moleculares para detectar SARS-CoV-2 no puede conocerse hasta que comprendamos quién está realmente infectado y quién no.

Hasta el momento el diagnóstico de laboratorio de COVID-19 se puede realizar mediante tres métodos. El recomendado por todas las agencias internacionales, e incuestionable, es la detección molecular de ARN viral en muestras respiratorias ${ }^{(5,6)}$. Luego encontramos los métodos rápidos de detección de antígeno del virus en muestras respiratorias y, por último, los métodos serológicos que buscan la respuesta de anticuerpos de tipo IgM, IgA, IgG o totales. Los diversos métodos presentan diferente sensibilidad y especificidad clínica. A su vez, en el proceso diagnóstico, esta performance depende de la calidad de la muestra y del día en que la misma se colecta, en relación al día de inicio de los síntomas.

\section{Detección molecular de SARS-CoV-2}

Como se citó anteriormente, la utilización de la detección molecular del ARN viral como prueba diagnóstica es incuestionable, dado su excelente desempeño analítico. Cabe destacar que la sensibilidad y especificidad analíticas de la mayoría de las técnicas de retrotrans- cripción seguida de reacción en cadena de la polimerasa (RT-PCR) son excelentes ${ }^{(7)}$.

Sin embargo, cuando se considera el proceso diagnóstico completo partiendo desde la recolección de la muestra, la sensibilidad clínica desciende en función del tipo y la calidad de muestra, el transporte y el almacenamiento de ésta, así como la etapa evolutiva de la enfer$\operatorname{medad}^{(8,9)}$.

Para las pruebas de diagnóstico molecular inicial de SARS-CoV-2, el CDC de Estados Unidos recomienda colectar y analizar muestras del tracto respiratorio superior. Estas muestras comprenden el hisopado nasofaríngeo $\mathrm{y} / \mathrm{o}$ orofaríngeo $\mathrm{y} / \mathrm{o}$ nasal, presentando el primero mejor sensibilidad y siendo el de preferencia ${ }^{(5)}$. Se pueden utilizar otras muestras como el aspirado nasofaríngeo, expectoración, aspirado traqueal y lavado bronquioloalveolar ${ }^{(5,6)}$.

\section{Preanalítica}

El proceso diagnóstico comienza con la indicación del estudio. Sin entrar en los distintos enfoques para indicar los estudios, entendemos que la indicación debe ser pautada por un consenso entre médicos prescriptores y profesionales del laboratorio clínico. Particularmente, en esta pandemia, han realizado un importante aporte los grupos de expertos Intercátedra de la Universidad de la República ${ }^{(10)}$, así como el MSP cumpliendo este último un rol importante en la articulación, coordinación y definición de pautas.

\section{- Recolección de muestra}

Este procedimiento, como fue citado, tiene un rol preponderante en el éxito de la prueba diagnóstica, por lo tanto su realización deberá cumplir con los mejores estándares de calidad. Para la recolección adecuada de la muestra debemos plantearnos tres elementos importantes.

1. Oportunidad. El virus del SARS-CoV-2 puede detectarse inicialmente en muestras de HNF uno a dos días antes del inicio de los síntomas ${ }^{(11,12)}$ y persiste durante siete a doce días en casos moderados ${ }^{(13)}$. Sin embargo, esto depende del curso clínico de la enfermedad, habiéndose reportado la persistencia de prueba positiva durante más de veinte días ${ }^{(13)}$. En pacientes graves se recomienda realizar toma de muestra de tracto respiratorio profundo si el primer estudio de HNF fue negativo ${ }^{(14)}$.

2. Calidad de la muestra. El HNF tiene un protocolo de recolección bien establecido y es un procedimiento molesto para el paciente, incluso puede provocar lagrimeo $^{(15)}$. No se puede usar cualquier hisopo. Debe ser flexible y su punta debe ser de poliéster con eje de material plástico. No estaì recomendado el uso hiso- 
pos de alginato de calcio, algodón o hisopos con ejes de madera, ya que pueden contener sustancias que inactivan virus o inhiben las pruebas moleculares ${ }^{(16)}$.

3. Transporte y almacenamiento. El hisopo obtenido se debe colocar en un tubo, de preferencia, con medio de transporte viral (MTV). Las alternativas para el transporte serían: soluciones tamponadas (PBS) o suero fisiológico $(\mathrm{SF})^{(17)}$. Si se colecta muestra orofaríngea (OF), ambos hisopos (HNF y OF) deben combinarse en un solo tubo con líquido de transporte. Al colocar los hisopos dentro de los tubos, se debe cortar el eje del hisopo, utilizando hisopos troquelados, o a través del uso de material cortante estéril o adecuadamente desinfectado entre recolecciones.

El aspirado nasofaríngeo (ANF) está especialmente indicado en niños menores de 2 años. Este se realiza con una sonda asociada a un frasco o tubo trampa. En este caso, se puede enviar el aspirado si se prevé un procesamiento antes de las 24 horas de recolectada la muestra, pero es recomendable luego de realizar el ANF propiamente dicho, aspirar medio de transporte viral al tubo trampa. De una manera similar se haría con otras muestras, como el aspirado traqueal o lavado bronquioloalveolar, indicados en pacientes sometidos a ventilación mecánica invasiva ${ }^{(4)}$. Las muestras colectadas de esta manera no pueden ser compartidas para uso de detección de otros virus respiratorios, salvo se efectúe en el propio laboratorio donde se realice las búsqueda de SARS-CoV-2 y se realicen alícuotas en forma segura.

Una vez que tenemos la muestra en el tubo, debemos asegurarnos que esté rotulado con los datos filiatorios del paciente. Muchos laboratorios utilizan etiquetas con código de barras. Para el transporte interno, el tubo se debe colocar en doble embolsado, mediando antisepsia de manos entre embolsado primario y secundario. Este transporte debería realizarse en recipiente rígido, cerrado con tapa, manteniendo el tubo en posición vertical. En el caso de muestras que deben salir fuera de la institución se recomienda el triple envase, reglamentado en el anexo 4 del decreto $129 / 005^{(18)}$. Las muestras deben transportarse al laboratorio a una temperatura entre $2^{\circ} \mathrm{C}$ y $8^{\circ} \mathrm{C}$, destacando que la la muestra debería procesarse antes de las 72 horas desde recolección, si se utiliza medio de transporte viral y dentro de las 24 horas de colectada si se utiliza SF o $\mathrm{PBS}^{(5)}$. Sin el medio de transporte o almacenamiento adecuado, las muestras se degradan. Esto es especialmente cierto para el ARN que se detecta mediante una prueba de RT-PCR. El ARN es menos estable que el ácido desoxirribonucleico (ADN), por lo que si una muestra no se transporta o almacena adecuadamente, aumenta el riesgo de un resultado RT-PCR falso negativo.
Una vez que la muestra llega al laboratorio, se realizan los controles habituales preanalíticos de la muestra. La conservación dentro del laboratorio también debe realizarse a temperatura entre $2{ }^{\circ} \mathrm{C}$ y $8{ }^{\circ} \mathrm{C}$ hasta poder iniciar el procesamiento. El procesamiento inicial (antes de la inactivación) de todas las muestras debe realizarse en cabina de bioseguridad, debidamente mantenida y validada $^{(19)}$.

\section{Analítica}

El procedimiento analítico dependerá de las características del laboratorio, de la capacitación del personal así como del equipamiento existente. La detección molecular comprende dos procedimientos: la extracción del ARN y posteriormente RT-PCR.

\section{- Extracción de ARN}

Esta parte del proceso analítico define, en muchas ocasiones, las características del flujo de trabajo del procedimiento completo. En base al equipamiento de extracción de ARN, podemos clasificar a los equipos automatizados en equipos de baja, mediana y alta capacidad de procesamiento en función de la cantidad simultánea de muestras capaz de procesar. Los equipos de baja capacidad presentan en general una respuesta rápida (aproximadamente tres horas) para un bajo número de muestras (una a cuatro muestras); sin embargo, los equipamientos de alta capacidad presentan un tiempo de procesamiento más prolongado (aproximadamente ocho horas) pero con número de muestras procesadas significativamente mayor (96 muestras). En general, en este último grupo el procesamiento se realiza en tandas, lo que implica juntar una determinada cantidad de muestras para luego iniciar el proceso analítico. Ante la carencia de insumos para algunos equipos de extracción de ARN automatizados, cabe señalar como una alternativa válida la utilización de procedimientos de extracción manual, aunque este tipo de metodología debe enmarcarse en un riguroso sistema de trazabilidad, capacitación del personal técnico así como la utilización de kits comerciales validados para este fin.

\section{- RT-PCR}

Este proceso comprende la etapa de retrotranscripción (RT) y la etapa de amplificación. La RT puede realizarse de forma separada o unida en un mismo paso a la segunda, según el protocolo o kit utilizado. Para comprender los diferentes protocolos utilizados en la etapa de la amplificación de SARS-CoV-2 debemos comprender el contenido genético del virus, el cual se observa en la figura 1. Desde el inicio de la circulación e identificación del SARS-CoV-2 se comenzaron los trabajos de puesta a punto de protocolos para la detección del virus en diferentes centros académicos y científicos del mundo. La 




OMS realizó una evaluación y recomienda la utilización de los protocolos que se observan en la tabla 1.

Los protocolos citados presentan diferentes objetivos moleculares, y a su vez los mismos tienen pequeñas diferencias en su sensibilidad analítica ${ }^{(20)}$. Sin embargo, todos muestran un muy buen desempeño para el diagnóstico de este virus. Si el límite de detección (LoD) para una prueba es demasiado alto, los pacientes infectados con SARS-CoV-2 pueden no dar positivo, lo que lleva a una alta tasa de resultados falsos negativos. Si el LoD para una prueba es demasiado bajo, entonces la contaminación puede convertirse en un problema importante, ya que la prueba detectará las cantidades más pequeñas de ARN viral, lo que conducirá a resultados positivos falsos (es decir, personas que no están infectadas con SARS-CoV- 2 darán positivo en lugar de negativo). El LoD está disponible en las instrucciones de uso de las pruebas de SARS-CoV-2 comerciales.

Aunque la recomendación para la confirmación de caso establece la detección de dos objetivos moleculares positivos (por ejemplo, N y RdRp o E y RdRp), la OPS ha recomendado que, una vez establecido el virus en la región o país, es suficiente la detección de un solo objetivo molecular para confirmar el diagnóstico ${ }^{(9)}$. Cabe advertir que si bien la mayoría de los objetivos moleculares presentan alta sensibilidad analítica, RdRp tiene una sensibilidad algo menor al resto, por lo que su utilización como único objetivo molecular debe desaconsejarse ${ }^{(20)}$. En Uruguay, investigadores de la Universidad de la República y del Instituto Pasteur desarrollaron una prueba de RT-PCR ${ }^{(21)}$. Esta prueba es comercializada por la empresa Atgen y detecta secuencias específicas del gen que codifica para la proteína $\mathrm{N}$ de la nucleocápside viral ${ }^{(22)}$.

Tabla 1. Protocolos de amplificación respaldados por Organización Mundial de la Salud.

\begin{tabular}{lll}
\hline País & Instituto & Genes objetivos \\
\hline China & China CDC & Orf1ab y N \\
Alemania & Charité & RdRP, E, N \\
Hong Kong & University of Hong Kong & Orf1b-nsp14, N \\
Japón & National Institute of & Pancorona y múltiples \\
& Infectious Diseases & dianas, S \\
Tailandia & National Institute of & N \\
& Health & \\
Estados Unidos & Center for Diseases & Tres dianas en el gen N \\
& Control & \\
Francia & Instituto Pasteur & Dos dianas en RdRP \\
\hline & & \\
\hline
\end{tabular}

\section{Posanalítica}

La interpretación de las pruebas moleculares se debe hacer con prudencia. A pesar de que se han revelado algunas correlaciones, las cargas virales determinadas por RT-PCR no deben utilizarse para indicar la gravedad de la infección o para controlar la respuesta terapéutica $^{(2,11,23)}$. Sin embargo, valores bajos de $\mathrm{Ct}$ (umbral de ciclo) indican altas cargas virales y pueden usarse como una indicación de mayor transmisibilidad ${ }^{(24)} . \mathrm{Ct}$ es la intersección entre una curva de amplificación y una línea de umbral. Es una medida relativa de la concentración del objetivo en la reacción de PCR en tiempo real. 
La interpretación de los resultados se debería realizar valorando el contexto clínico-epidemiológico que presenta el paciente, especialmente en aquellos que requieran internación. Los resultados negativos en un escenario de alta sospecha deben obligarnos a valorar las posibles causas de falsos negativos. Pueden aparecer falsos negativos en las siguientes circunstancias ${ }^{(8,9)}$.

- Recolección inadecuada del HNF.

- Retraso o pérdida de cadena de frío durante el transporte.

- Error preanalítico en el etiquetado de la muestra a lo largo del proceso.

- Poca eliminación de virus por el paciente dado el estadio de la infección o por la gravedad de ésta.

- Presencia de mutaciones del virus en las regiones a las que se dirigen los ensayos.

Además de estos aspectos preanalíticos también puede haber factores analíticos relacionados con la aparición de falsos negativos. En ese sentido, la sensibilidad podría verse afectada por mutaciones virales en los sitios objetivo, así como por la presencia de inhibidores presentes en la muestra. Ambas situaciones, hasta el momento, fueron muy poco frecuentes. Sin embargo, en un estudio que analizó 373 secuencias genéticas de virus SARS-CoV-2 de Latinoamérica, incluyendo 11 de Uruguay, se demostró la presencia de cambios en las regiones del gen $\mathrm{N}$ que podrían impedir un diagnóstico molecular preciso ${ }^{(25)}$. En cambio, la región del gen E parece mantenerse conservada, por lo que los autores la recomiendan como candidata ideal para la detección de primera línea en Latinoamérica ${ }^{(25)}$. En Europa, por el contrario, ya se ha detectado falla en la detección del gen E debido a una mutación ${ }^{(26)}$. Por tanto, a pesar de las recomendaciones de la OPS de investigar un objetivo único, a la luz de estos trabajos resulta riesgoso confiar en un ensayo dirigido a una única secuencia del genoma viral. También se pone de manifiesto la necesidad de mantener la vigilancia, a través de secuenciación completa, de SARS-CoV-2 con el objetivo de buscar variantes que interfieran con la capacidad de detección de las pruebas de RT-PCR. Finalmente, sería recomendable que los fabricantes fueran transparentes sobre los cebadores y las sondas utilizadas, ya que esto permitiría que las variantes problemáticas se identificaran más fácilmente a partir de las secuencias virales disponibles.

En los ensayos moleculares el hallazgo de falsos positivos es muy poco frecuente. Estos podrían deberse a error preanalítico o contaminación cruzada entre muestras durante el procesamiento analítico ${ }^{(8)}$.

Luego de la interpretación de los resultados, se realiza el informe escrito con los resultados obtenidos y la firma del profesional que se responsabiliza por los resul$\operatorname{tados}^{(27)}$.

\section{Detección de antígenos virales}

Al día de estar realizando este manuscrito, estas pruebas no están disponibles en nuestro país. Sin embargo, el desarrollo de anticuerpos monoclonales contra la proteína de nucleocápside es la base de los métodos rápidos de detección de antígenos de SARS CoV-2 en muestras respiratorias $^{(28)}$.

En un estudio, aún sin revisión de pares, este tipo de ensayo mostró una sensibilidad de $50 \%$ cuando la RT-PCR fue positiva y de $82,2 \%$ frente a un $\mathrm{Ct}<25$. La especificidad reportada fue de $100 \%{ }^{(29)}$. La SEIMC emitió recomendaciones señalando que los ensayos preliminares realizados con este tipo de pruebas, disponibles en España, mostraron una sensibilidad inferior a $30 \%$ y una especificidad de $100 \%$. La Sociedad afirmó que estos resultados impedirían su introducción en la rutina ${ }^{(8)}$. Por otra parte, desconocemos la cinética de detección de antígenos, presuponemos que sería similar a la del ARN viral ${ }^{(9)}$. Por tanto, a pesar de los prometedores resultados de algunos trabajos, cualquier tipo de prueba de este tipo que se fuera a comercializar debería pasar por un proceso de verificación con muestras respiratorias. Si se verifica su alta especificidad podría utilizarse en aquellos pacientes sintomáticos que requieran internación. Por otro lado, dada la sensibilidad reportada, solo sería de utilidad en aquellos casos que diera positiva. Es claro que si esta prueba da negativa, se debe realizar el ensayo molecular.

Las ventajas de este tipo de ensayo es que se trataría de pruebas rápidas que se podrían realizar en cualquier laboratorio en condiciones de bioseguridad adecuadas $^{(8)}$.

\section{Detección de respuesta inmune}

\section{Introducción}

Generalmente en las infecciones virales, alrededor del inicio de los síntomas, los leucocitos inician el proceso de fabricar anticuerpos contra diferentes estructuras virales. Al principio estos anticuerpos, de tipo IgM, son bastante inespecíficos, lo cual podría dar lugar a reacciones cruzadas. Estos anticuerpos son transitorios y se tornan indetectables luego de algunas semanas. Los anticuerpos de tipo IgA, característicos de las secreciones mucosas, tienen una cinética de aparición similar a los $\operatorname{IgM}^{(30)}$. A lo largo del tiempo la respuesta inmune madura, se hace más específica contra antígenos propios de SARS CoV-2. Igual hay posibilidad que estos anticuerpos de tipo $\operatorname{IgG}$, aún, reaccionen contra antígenos presentes en la misma familia de virus. Sin embargo, la aparición de IgG indica que el paciente se ha infectado 
con el virus y ha montado una respuesta inmune contra éste. Solo una publicación realizada en modelo animal y aún sin revisión de pares, establece que la respuesta inmune protege contra la reinfección ${ }^{(31)}$. Por tanto, aún falta evidencia para afirmar que la respuesta inmune es protectora.

\section{Pruebas serológicas}

Las pruebas serológicas que identifican IgA, IgM, IgG o incluso anticuerpos totales contra SARS-CoV-2 se realizan en muestras de sangre, suero o plasma. Estas pruebas son menos complejas de realizar que las pruebas moleculares. Sin embargo, no son útiles para diagnosticar infecciones en etapa aguda ${ }^{(32)}$. La respuesta de anticuerpos a la infección tarda un par de semanas en ser detectables de manera confiable ${ }^{(30)}$. Los resultados negativos no excluyen la infección por SARS-CoV-2, particularmente en pacientes con exposición reciente al virus. Existen estudios publicados que utilizaron sustratos antigénicos variados y elaborados en cada laboratorio. Estos incluyeron: proteína de nucleocápside $(\mathrm{N})^{(29)}$, combinación de proteína $\mathrm{N}$ más proteínas de espícula ${ }^{(23)}$ y otros utilizaron solo proteínas de espícula ${ }^{(32)}$.

Para valorar la especificidad de los ensayos serológicos, el estudio de Guo y colaboradores ${ }^{(30)}$ utilizó plasmas con anticuerpos contra los coronavirus humanos -229E, -NL63, -OC43, -HKU1, y SARS-CoV. La prueba serológica para SARS-CoV-2 solo mostró reactividad cruzada con el plasma que tenía anticuerpos contra SARS-CoV, pero no hubo reactividad frente a los otros coronavirus humanos ${ }^{(30)}$. Quitando esta situación la prueba demostró alta especificidad. En cuanto a la sensibilidad, IgM mostró $76 \%$ de sensibilidad al día 7 desde inicio de síntomas, en casos confirmados por método molecular. La media de aparición de IgM fue el día 5 desde inicio de los síntomas con un intervalo intercuartílico de 3-6 días. La IgG mostró una sensibilidad de 78\%. La media de aparición de IgG fue el día 14 desde el inicio de los síntomas con un intervalo intercuartílico de 10-18 días. En el estudio de Zhao J y colaboradores ${ }^{(32)}$ la media de aparición de IgM fue el día 12 y mostró una sensibilidad de $82,7 \%$, con gran variación en los días de aparición de respuesta. En el caso de $\operatorname{IgG}$ la media de aparición fue el día 14 con una sensibilidad de 64,7\%.

\section{Tipos de pruebas serológicas comercializadas}

En cuanto a los tipos de pruebas comercializados, hasta el momento, a nivel mundial, se han comercializado tres tipos de pruebas.

- Ensayos de inmunoabsorción ligado a enzimas (ELISA).
- Pruebas rápidas que detectan $\operatorname{IgM} / \operatorname{IgG}$ en soporte inmunocromatográfico.

- Ensayos automatizados por quimioluminiscencia.

Hasta el 24 de junio de 2020, la US Food and Drug Administration (FDA) ha emitido autorizaciones de uso de emergencia (EUA) a varias pruebas serológicas. Mayoritariamente se trata de ensayos automatizados por quimioluminiscencia. Pero también ensayos de ELISA y pruebas rápidas que detectan $\operatorname{IgM}$ e $\operatorname{IgG}$ (33). EUA implica que la empresa comercial ha enviado a FDA información sobre el desempeño del ensayo para su revisión y aprobación.

El FIND, a través de varias instituciones colaboradoras, evaluó varios ensayos rápidos de detección de $\mathrm{IgM} / \mathrm{IgG}$ obteniendo resultados muy variables en relación tanto a sensibilidad como especificidad ${ }^{(34)}$.

Dados estos hallazgos se hace necesario evaluar los ensayos a comercializar en Uruguay para conocer su desempeño analítico y clínico.

Si contáramos con ensayos serológicos con un buen desempeño, serían de utilidad cuando los pacientes consultan con complicaciones tardías de la enfermedad, en casos de RT-PCR falsamente negativa, dado que la eliminación viral disminuye con el tiempo ${ }^{(8)}$.

Por otra parte, ensayos serológicos que evalúen con precisión la infección previa y la inmunidad al SARS-CoV-2 serán esenciales para los estudios epidemiológicos, la vigilancia continua, los estudios de vacunas y potencialmente para la evaluación del riesgo de los trabajadores de la salud ${ }^{(8,35)}$.

\section{Conclusiones}

Al día de hoy el diagnóstico de COVID-19 se debe realizar a través de métodos moleculares, vigilando el cumplimiento de la etapa preanalítica con la finalidad de minimizar los falsos negativos. Las técnicas que detectan antígenos son prometedoras, pero falta evaluación local. La pruebas serológicas que lograran un desempeño satisfactorio serán de utilidad para pacientes con síntomas respiratorios pero que no consultan en la fase inicial o aguda. $Y$, en un futuro, van a ser fundamentales para conocer el estatus inmunitario de la población.

\section{Summary}

Over 500 COVID-19 diagnostic tests are available in the global market or are completing the final stages of development. Within this unprecedented framework, it is important to learn about the different types of trials available and understand their role in the diagnostic process. An accurate diagnosis is key for the handling of the pandemic, since it allows for the right identifica- 
tion of cases and thus entitles authorities to take the subsequent control measures: search for contacts and isolation. This review describes the foundations and usefulness of the different types of etiological diagnosis. It also establishes the pre-analytical conditions required to apply them, their clinical sensitivity and specificity, as well as the right interpretation and issuance of results.

\section{Resumo}

Mais de 500 testes ou exames diagnósticos para COVID-19 estão sendo comercializados no mercado mundial ou estão em estágio avançado de desenvolvimento. Nesta situação sem precedentes, é importante compreender o tipo de ensaios disponíveis, bem como o seu papel no processo diagnóstico. O diagnóstico preciso é fundamental para controlar a pandemia. Isso permite a identificação adequada dos casos, o que possibilita medidas de controle subsequentes: busca de contatos e isolamento. Nesta revisão, descrevemos a lógica e a utilidade dos diferentes tipos de testes diagnósticos etiológicos disponíveis. Também estabelecemos as condições pré-analíticas necessárias para sua realização, a sensibilidade e especificidade clínicas, bem como a correta interpretação e a liberação dos resultados.

\section{Bibliografía}

1. Foundation for Innovative New Diagnostics. SARSCOV-2 diagnostic pipeline. Disponible en: https://www.finddx.org/covid-19/pipeline/?avan$\mathrm{ce}=$ all\&type $=$ all\&status $=$ all\&section $=$ show-all\&action $=$ default [Consulta: 18 abril 2020].

2. Liu Y, Yan LM, Wan L, Xiang TX, Le A, Liu JM, et al. Viral dynamics in mild and severe cases of COVID-19. Lancet Infect Dis 2020; 20(6):656-7. doi: 10.1016/S14733099(20)30232-2.

3. Patel R, Babady E, Theel ES, Storch GA, Pinsky BA, St George K, et al. Report from the American Society for Microbiology COVID-19 International Summit, 23 March 2020: value of diagnostic testing for SARS-CoV-2/ COVID-19. mBio 2020;11(2):e00722-20.

4. Saah AJ, Hoover DR. "Sensitivity" and "specificity" reconsidered: the meaning of these terms in analytical and diagnostic settings. Ann Intern Med 1997; 126:91-4 doi: 10.7326/0003-4819-126-1-199701010-00026.

5. Center for Diseases Control and Prevention. Information for laboratories about coronavirus (COVID-19). Disponible en: https://www.cdc.gov/coronavirus/2019-nCoV/lab/index.html [Consulta: 15 abril 2020].

6. World Health Organization. Laboratory testing for coronavirus disease 2019 (COVID-19) in suspected human cases: interim guidance, 2 March 2020. Disponible en: https://apps.who.int/iris/handle/10665/331329 [Consulta: 15 abril 2020].
7. Foundation for Innovative New Diagnostics. SARSCoV-2 molecular assay evaluation: results. Disponible en: https://www.finddx.org/wp-content/uploads/2020/ 05/FIND_SARS-COV2 molecular-assay-evaluation-results_15May2020.pdf [Consulta: 27 mayo 2020].

8. Grupo de expertos SEIMC para el análisis del diagnóstico microbiológico del COVID-19. Recomendaciones institucionales documento de posicionamiento de la SEIMC sobre el diagnóstico microbiólogo de COVID-19. SEIMC, 2000:7p. Disponible en: https://seimc.org/contenidos/documentoscientificos/recomendaciones/seimc-rc-2020-Posicionamiento_SEIMC_diagnostico_microbiologico_COVID19. pdf [Consulta: 27 mayo 2020].

9. Organización Panamericana de la Salud. Directrices de laboratorio para la detección y el diagnóstico de la infección con el virus responsable de la COVID-19. Disponible en: https://www.paho.org/en/documents/laboratory-guidelines-detection-and-diagnosis-covid-19-virus-infection [Consulta: 22 abril 2020].

10. UdelaR. Facultad de Medicina. Cátedra de Enfermedades Infecciosas. Actualización. Recomendaciones grupo interdisciplinario inter sociedades científicas e inter cátedras de Uruguay. Versión de 24 de marzo de 2020. Disponible en: http://www.infectologia.edu.uy/divulgacion-medica/novedades-y-avances/actualizacion-recomendaciones-intersociedades-y-catedras [Consulta: 22 abril 2020].

11. Pan Y, Zhang D, Yang P, Poon LLM, Wang Q. Viral load of SARS-CoV-2 in clinical samples. Lancet Infect Dis 2020; 20(4):411-2. doi: 10.1016/S1473-3099(20)30113-4.

12. Wei WE, Li Z, Chiew CJ, Yong SE, Toh MP, Lee VJ. Presymptomatic transmission of SARS-CoV-2 - Singapore, January 23-March 16, 2020. MMWR Morb Mortal Wkly Rep 2020; 69:411-5. doi: 10.15585/mmwr.mm6914e1.

13. Wölfel R, Corman VM, Guggemos W, Seilmaier M, Zange S, Müller MA, et al. Virological assessment of hospitalized patients with COVID-2019. Nature 2020; 581:465-9. doi: 10.1038/s41586-020-2196-x.

14. European Centre for Disease Prevention and Control. Questions and answers regarding laboratory topics on SARS-CoV-2. Disponible en: https://www.ecdc.europa.eu/en/all-topics-z/coronavirus/threats-and-outbreaks/covid-19/laboratory-support/questions [Consulta: 23 abril 2020].

15. Marty FM, Chen K, Verrill KA; New England Journal of Medicine. Videos en Clinical Medicine. Performen medical procedures: how to obtain a nasopharyngeal swab specimen. 2020. Disponible en: https://www.youtube.com/watch ?v=1jVNeSnVSks [Consulta: 23 abril 2020].

16. Center for Diseases Control and Prevention. Interim guidelines for collecting, handling, and testing clinical specimens from persons for coronavirus disease 2019 (COVID-19). Disponible en: https://www.cdc.gov/coronavirus/2019-nCoV/lab/guidelines-clinical-specimens.html [Consulta: 15 abril 2020].

17. Rodino KG, Espy MJ, Buckwalter SP, Walchak RC, Germer JJ, Fernholz E, et al. Evaluation of saline, phospha- 
te-buffered saline and minimum essential medium as potential alternatives to viral transport media for SARS-CoV-2 testing. J Clin Microbiol May 2020; 58(6):e00590-20. doi: 10.1128/JCM.00590-20.

18. Uruguay. Poder Ejecutivo. Decreto $\mathrm{N}^{\circ} 382 / 014$ de 24/12/2014 artículo 1. Disponible en: https://www.impo. com.uy/bases/decretos-reglamento/382-2014/1 [Consulta: 15 abril 2020].

19. World Health Organization. Laboratory biosafety guidance related to the novel coronavirus (2019-nCoV). Interim guidance 12 February 2020. Disponible en: https://www. who.int/docs/default-source/coronaviruse/laboratory-biosafety-novel-coronavirus-version-1-1.pdf?sfvrsn=912a9847_2 [Consulta: 15 abril 2020].

20. van Kasteren PB, van der Veer B, van den Brink S, Wijsman $\mathrm{L}$, de Jonge J, van den Brandt $A$, et al. Comparison of seven commercial RT-PCR diagnostic kits for COVID-19. J Clin Virology 2020; 128:104412. doi: 10.1016/j.jcv. 2020.104412 .

21. Moreno P, Moratorio GA, Iraola G, Fajardo A, Aldunate F, Pereira M, et al. An effective COVID-19 response in South America: the uruguayan conundrum. MedRxiv 2020.07.24.20161802. doi: 10.1101/2020.07.24.20161802.

22. UdelaR; Institut Pasteur. COVID-19 RT-PCR Real TM Fast (HEX). Disponible en: http://www.atgen.com.uy/sitio/wp-content/uploads/2020/05/Inserto-71-v2-HEX.pdf [Consulta: 10 agosto 2020].

23. To KK, Tsang OT, Leung WS, Tam AR, Wu TC, Lung DC, et al. Temporal profiles of viral load in posterior oropharyngeal saliva samples and serum antibody responses during infection by SARS - CoV -2: an observational cohort study. Lancet Infect Dis 2020; 20:565-74. doi: 10.1016/S1473-3099(20)30196-1.

24. Cheng V, Wong S, Chen J, Yip C, Chuang V, Tsang O, et al. Escalating infection control response to the rapidly evolving epidemiology of the coronavirus disease 2019 (COVID-19) due to SARS-CoV-2 in Hong Kong. Infect Control Hosp Epidemiol 2020; 41(5):493-8. doi: 10.1017/ ice.2020.58.

25. Ramírez JD, Muñoz M, Hernández C, Florez C, Gomez S, Rico A, et al. Genetic diversity among SARS-CoV2 strains in South America may impact performance of molecular detection. Pathogens 2020; 9:580. doi: 10.3390/pathogens 9070580 .

26. Artesi M, Bontems S, Göbbels P, Franckh M, Maes P, Boreux $R$, et al. A recurrent mutation at position 26,340 of
SARS-CoV-2 is associated with failure of the E-gene qRT-PCR utilized in a commercial dual-target diagnostic assay. J Clin Microbiol 2020; 58(10):e01598-20. doi: 10.1128/JCM.01598-20.

27. Hawkins R. Managing the pre- and post- analytical phases of the total testing process. Ann Lab Med 2012; 32:5-16. doi: 10.3343/alm.2012.32.1.5

28. Diao B, Wen K, Chen J, Liu Y, Yuan Z, Han C, et al. Diagnosis of Acute Respiratory Syndrome Coronavirus 2 Infections by detection of nucleocapsid protein. MedRxiv 2020.03.07. doi: 10.1101/2020.03.07.20032524.

29. Lambert-Niclot S, Cuffel A, Le Pape S, Vauloup-Fellous C, Morand-Joubert L, Roque-Afonso AM, et al. Evaluation of a rapid diagnostic assay for detection of SARS-CoV-2 antigen in nasopharyngeal swabs. J Clin Microbiol 2020; 58(8):e00977-20. doi: 10.1128/JCM.00977-20.

30. Guo L, Ren L, Yang S, Xiao M, Chang D, Yang F, et al. Profiling early humoral response to diagnose novel coronavirus disease (COVID-19). Clin Infect Dis 2020; 71(15): 778-85. doi: 10.1093/cid/ciaa310.

31. Bao L, Deng W, Gao H, Xiao C, Liu J, Xue J, et al. Reinfection could not occur in SARS-CoV-2 infected rhesus macaques. bioRxiv 2020.03.13.990226. doi: 10.1101/2020.03. 13.990226.

32. Zhao J, Yuan Q, Wang H, Liu W, Liao X, Su Y, et al. Antibody responses to SARS-CoV-2 in patients of novel coronavirus disease 2019. Clin Infect Dis 2020; ciaa344. doi: $10.1093 / \mathrm{cid} / \mathrm{ciaa3} 44$.

33. Food and Drug Administration. In vitro diagnostics EUAs. Emergency use authorization. Disponible en: https://www. fda.gov/medical-devices/emergency-situations-medical-devices/emergency-use-authorizations. [Consulta: 23 junio 2020].

34. Foundation for Innovative New Diagnostics. SARS-CoV-2 diagnostics: performance data. Disponible en: https://www.finddx.org/covid-19/dx-data/ [Consulta: 27 mayo 2020].

35. España. Ministerio de Sanidad. Instituto de Salud Carlos III. Interpretacioìn de las pruebas diagnoìsticas frente a SARS-CoV-2. 24 de abril de 2020. versioìn 2. Disponible en: https://www.mscbs.gob.es/profesionales/saludPublica/ccayes/ alertasActual/nCovChina/documentos/INTERPRETACION DE_LAS_PRUEBAS.pdf. [Consulta: 23 junio 2020].

\section{Contribución de autores}

Pablo López Pedrozo, https://orcid.org/0000-0002-6673-7131. Concepción, revisión de bibliografía y redacción.

Raquel Ballesté https://orcid.org/0000-0001-9699-8714. Concepción, diseño, revisión y redacción.

Verónica Seija, https://orcid.org/0000-0002-3585-9259. Concepción, diseño, revisión de bibliografía y redacción. 